\title{
Sirenomelia- The Mermaid Syndrome: A Case Report
}

\author{
Naveena.$S^{1}$, Mrudula $C^{2}$. \\ ${ }^{1,2}$ Assistant Professor, MD, Department of Anatomy, Apollo institute of Medical Sciences and Research, \\ Hyderabad
}

\begin{abstract}
Sirenomelia, Mermaid syndrome, is a very rare fatal congenital anomaly of sirenomelia sequence with fusion and atrophy of lower limbs, giving an appearance of human head and upper body with tail of a fish. The spectrum of anomalies affects primarily musculoskeletal system with severe urogenital and gastrointestinal malformations, neural tube and vertebral anomalies. It has a reported incidence of 1 in 60,000 births with unknown etiology. Depending on the degree of fusion of lower limb and development of feet sirenomelia is distinguished in 3 forms- sympus dipus, sympus monopus and sympus apus. Here we present a case of sirenomelia with sympus monopus, severe oligohydramnios and is still born. We examined the foetus in light of data from medical literature and discussed the embryological origin of such severe form of caudal dysgenesis.
\end{abstract}

Keywords: Caudal dysgenesis, Oligohydramnios, , Sirenomelia, Sympus dipus, Sympus monopus, Sympus apu, .

\section{Introduction :}

In Greek mythology, the Sirens were three creatures with the head of a woman and the body of a bird from the wings down. Over time, these bird-women were portrayed as more aquatic creatures, and eventually with a full mermaid-like appearance.

Sirenomelia sequence, also known as sirenomelia, is a birth defect of the lower body characterized by the apparent fusion of the legs into a single lower limb. This is associated with a variable combination of severe visceral abnormalities, most commonly urogenital and gastrointestinal. It is incompatible with life.

The incidence of this condition is 1 in nearly 60,000 live births. The etiology of this is unknown . Maternal drug use, Rheumatoid factor ,maternal diabetes are identified as the common factors . It may result from a defect, at the late gastrula stage, in the formation or remodeling of the early embryonic vasculature or in the generation of the mesodermal precursors during the third gestational week.

Among other abnormalities that are commonly observed in sirenomelia are lumbosacral and pelvic malformations, including sacral agenesis, malformed vertebrae and hemivertebrae and corresponding anomalies of the central nervous system. However sirenomelia is also associated with malformations of the upper part of the body, including cleft palate, upper thoracic and cervical vertebra abnormalities, pulmonary hypoplasia, and cardiac defects.

With so many features in view in this paper we reported on the clinical and pathological features of sirenomelia from the fetus which we received in our institution Apollo institute of medical sciences and research and reviewed the literature. We did the dissection and identified the internal and external changes and presented the paper.

\section{Materials \& Methods :}

A 25-year-old diabetic with an unsupervised pregnancy of 28 weeks was admitted with severe abdominal pain in BBR Hospital ,Secunderabad. History of the patient tells that she is gravida 2 and had previous history of one abortion. Until that date, the concealed pregnancy was uneventful except for the woman's poorly controlled diabetes mellitus, and a history of moderate exposure to tobacco. There was no declared exposure to other drugs.

Ultrasonography of the case revealed a dead fetus, oligohydramnios and renal agenesis, severe caudal malformation, including fused lower extremities. The detailed evaluation was not possible because of the presence of severe oligohydramnios. The pictures of ultrasound were not included in this case report .

The female underwent vaginal delivery of a dead, malformed female fetus of $2000 \mathrm{gms}$.

\section{Observations :}

The stillborn fetus had Potter's face, fused lower limbs , anal opening on the posterior aspect of caudal end and with small tail like projection. 


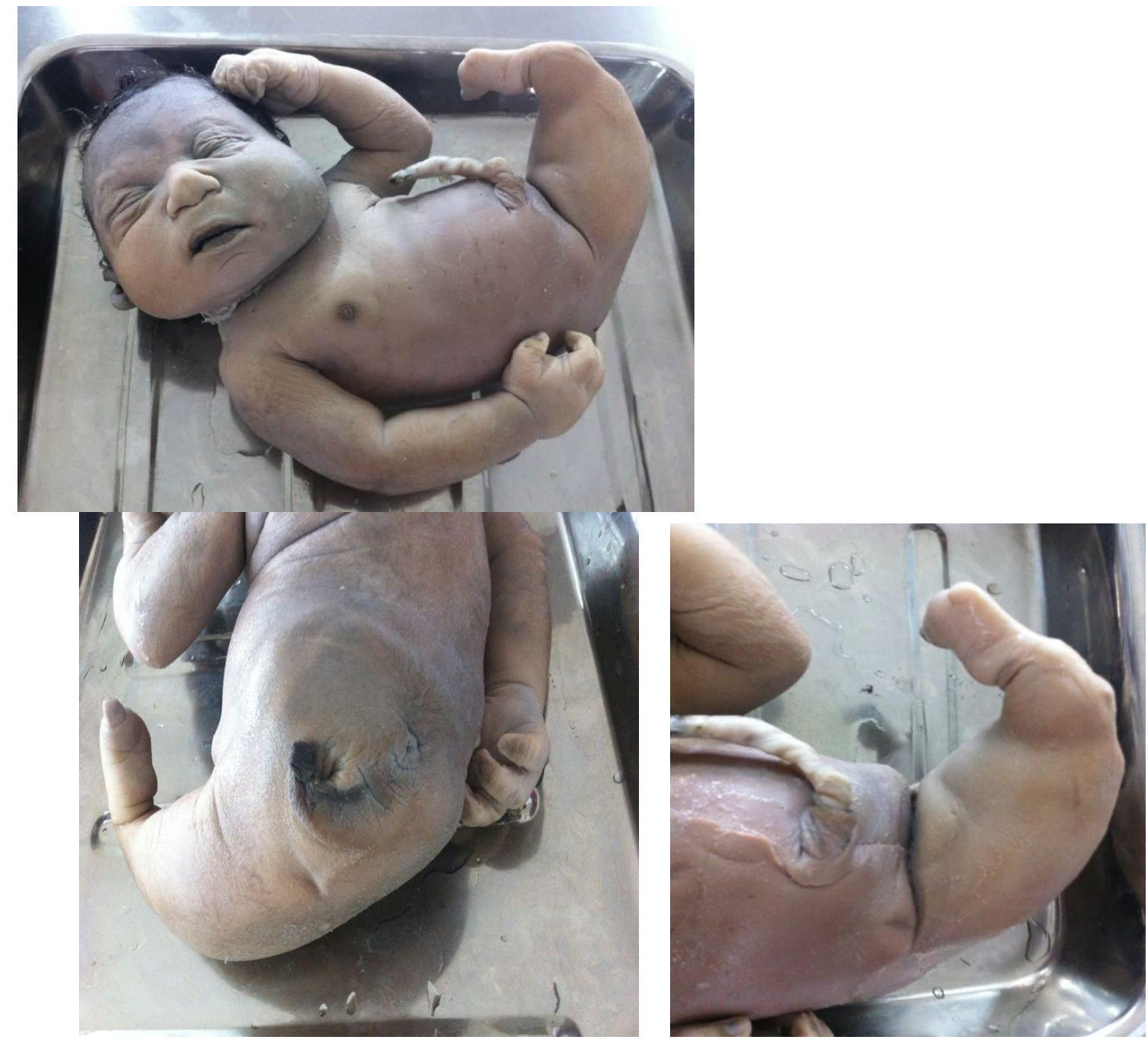

Autopsy confirmed the prenatal diagnosis of various features.

The thoracic region showed hypoplastic lungs on both sides, heart was normal in position with thymus in the anterior mediastinum region.
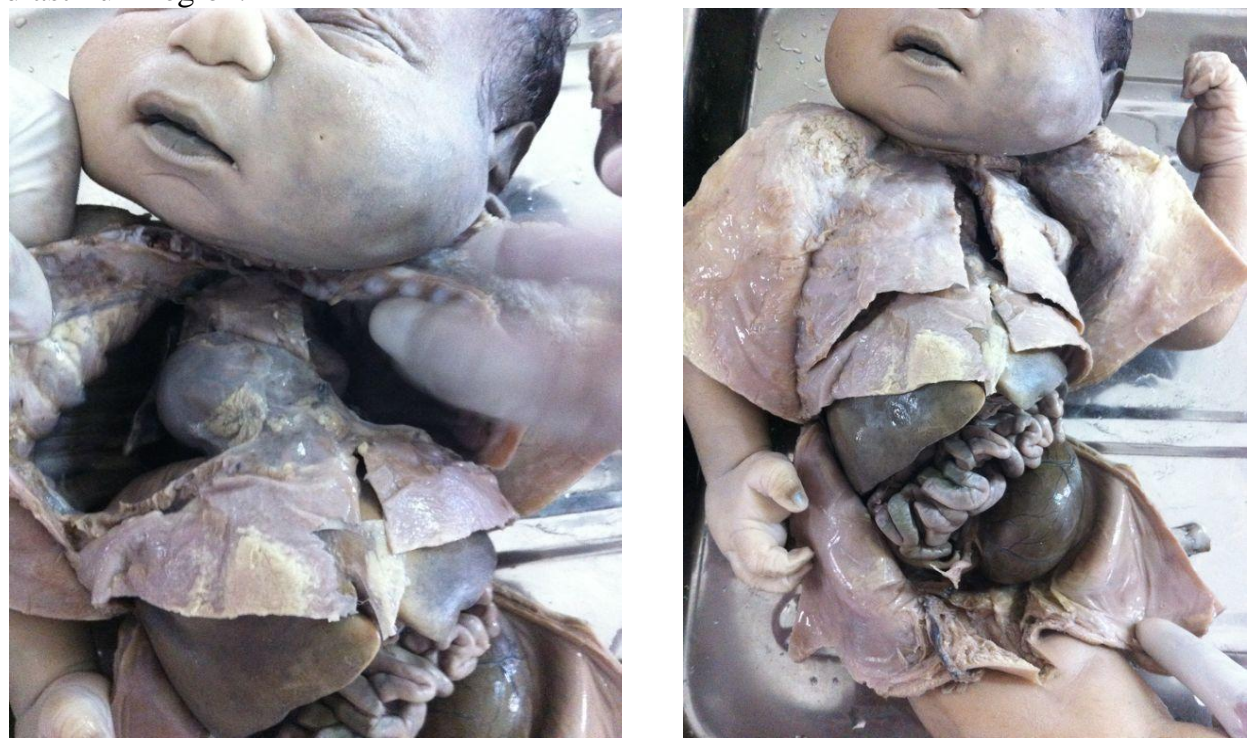

Abdominal dissection revealed renal agenesis on the left side, right kidney presented with lobulation, polycystic ovary in the right inguinal area, absence of rectum, anal canal and the terminal portion of the 
sigmoid colon full of meconium. Externally there is opening of the anal canal but internally no communication with the part of gastrointestinal tract. The uterus is absent and the fallopian tube is opening into the anal orifice. The abdominal aorta was dividing at the higher level into right and left common iliac arteries. The other abdominal organs are at normal position and in normal structure.

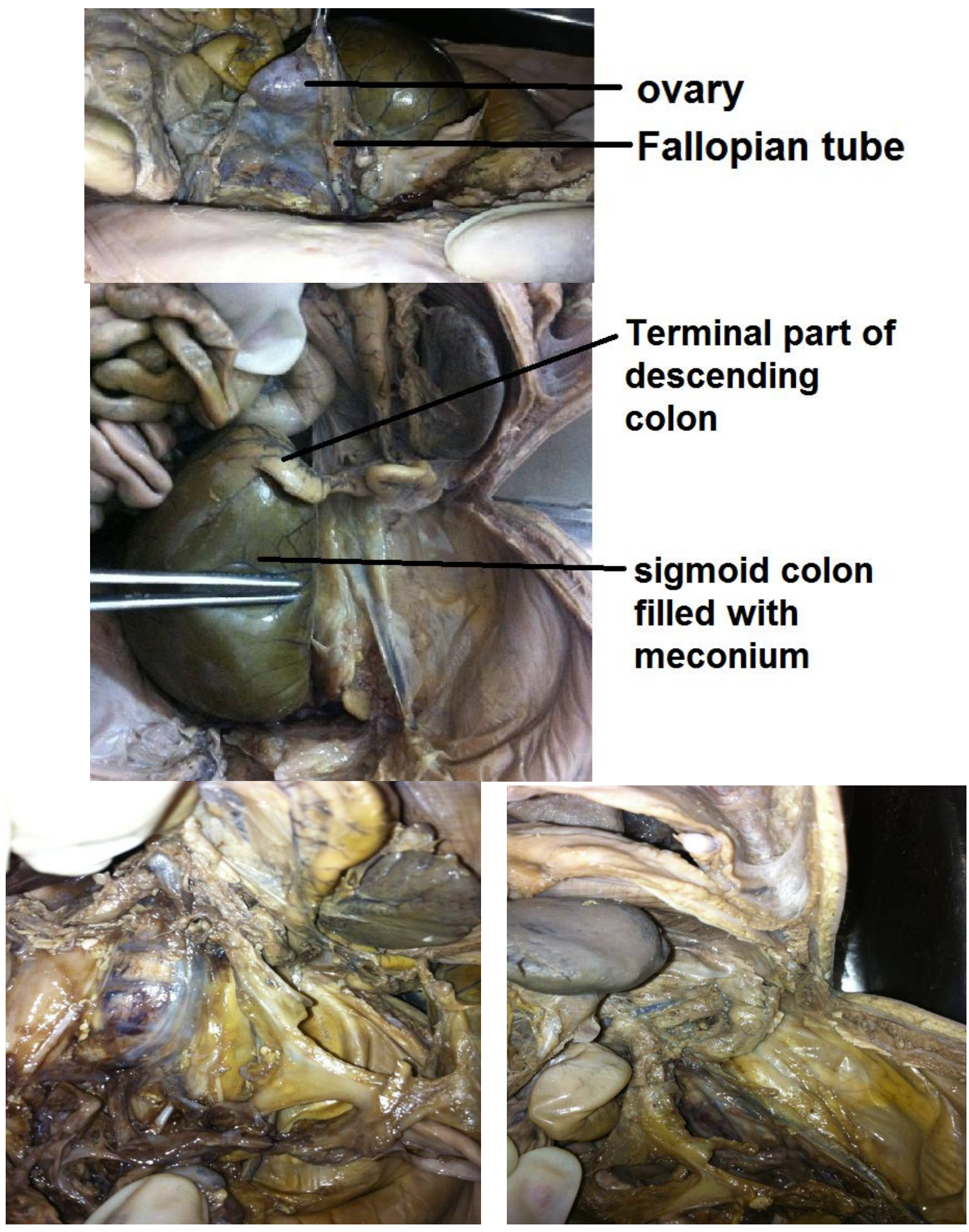

IV. Discussion :

The primary molecular defect underlying sirenomelia is unknown, clinical studies have given rise to several hypotheses to explain the causal mechanisms. The two main pathogenic hypotheses are the vascular steal hypothesis and the defective blastogenesis hypothesis. Other mechanical hypotheses that have been 
proposed to cause sirenomelia, such as lateral compression of the caudal body by amniotic folds and medial compression by overdistension of the neural tube.

According to the vascular steal hypothesis, fusion of the limbs results from a deficient blood flow and nutrient supply to the caudal mesoderm, which in turn would result in agenesis of midline structures and subsequent abnormal approximation of both lower limb fields. Vascular mechanisms have been proposed to underlie the pathogenesis of several other defects as well as sirenomelia (Sadler and Rasmussen, 2010).

According to the defective blastogenesis hypothesis, sirenomelia is a primary defect of blastogenesis that occurs during the final stages of gastrulation at the tailbud stage, corresponding to the third gestational week in humans (Opitz et al., 2002; Duesterhoeft et al., 2007; Davidson, 1993). The phenotypic variability depends on the intensity, time of initiation and duration of the underlying event.

Retinoic acid, maternal diabetes and heavy metals have been described as important environmental risk factors for caudal malformations. Kochhar (Kochhar, 1967) and Yasuda et al. (Yasuda et al., 1990) were the first to report that excess administration of RA to pregnant mice could lead to caudal malformations in the offspring, . Maternal diabetes is considered as a causative environmental factor for CD because $10-15 \%$ of affected children have diabetic mothers (Assimakopoulos et al., 2004; Castori et al., 2010). Exposure to heavy metals is associated with CD and sirenomelia in both experimental models (Hilbelink and Kaplan, 1986) and in

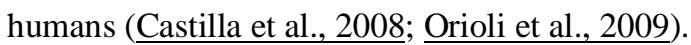

\section{Conclusion :}

Sirenomelia is a multisystemic human malformation of unknown etiology. Clinical observations over the years have provided important insights into the problem and given rise to causal hypotheses that can now be tested and analyzed at the molecular level owing to the availability of animal models. Much work remains to be done to define the precise role of the candidate factors in causing sirenomelia malformations. The future should include careful analyses of the role of Bmp and RA signaling pathways, as well as their potential interaction, in the generation of this malformation.

Clinical and basic research opportunities

- To determine the genetic roots of sirenomelia.

- To characterize the involvement of abnormal caudal mesoderm formation and vasculogenesis in sirenomelia.

- To obtain a better understanding of the role of the RA and Bmp signaling pathways in the development of sirenomelia.

\section{Acknowledgment:}

We cordially wish to express my acknowledgement to the family of BBR Hospital, Hyderabad and the parents who donated the foetus for the study. We will be failing badly if we don't mention the encouragement and support which we received from Apollo Institute Of Medical Sciences And Research, Hyderabad in doing the foetal dissection which has been our unique experience.

\section{References:}

[1]. Moosa S, Lambie LA, Krause A.Clin Dysmorphol. 2012 Jul;21(3):124-30. Sirenomelia: four further cases with discussion of associated upper limb defects.

doi: $\quad$ 10.1097/MCD.0b013e328354e51b. ;

[2]. Pillay M, Yesodharan D, Narayanan DL, Jojo A, Luiz N, Nampoothiri S. Pediatr Dev Pathol. 2012 Sep-Oct;15(5):403-6. doi: 10.2350/12-05-1199-CC.1. Epub 2012 Jul 20.; Sirenomelia: case reports and current concepts of pathogenesis.

[3]. Thottungal AD, Charles AK, Dickinson JE, Bower C. Am J Med Genet A. 2010 Oct;152A(10):2578-87.

[4]. doi: 10.1002/ajmg.a.33599.; Caudal dysgenesis and sirenomelia-single centre experience suggests common pathogenic basis.

[5]. Sikandar R, Munim S. ; J Pak Med Assoc. 2009 Oct;59(10):721-3.; Sirenomelia, the Mermaid syndrome: case report and a brief review of literature.

[6]. Rougemont AL, Bouron-Dal Soglio D, Désilets V, Jovanovic M, Perreault G, Laurier Oligny L, Fournet JC. ; Am J Med Genet A. 2008 Jun 1;146A(11):1470-6. doi: 10.1002/ajmg.a.32081.; Caudal dysgenesis, sirenomelia, and situs inversus totalis: a primitive defect in blastogenesis.

[7]. Onyeije CI, Sherer DM, Handwerker S, Shah L ; Am J Perinatol. 1998 Mar;15(3):193-7.; Prenatal diagnosis of sirenomelia with bilateral hydrocephalus: report of a previously undocumented form of VACTERL-H association. 